\title{
DETERMINANTS OF HEAVY SMOKING: RESULTS FROM THE GLOBAL ADULT TOBACCO SURVEY IN POLAND (2009-2010)
}

\section{DOROTA KALETA ${ }^{1}$, TERESA MAKOWIEC-DĄBROWSKA ${ }^{2,3}$, ELŻBIETA DZIANKOWSKA-ZABORSZCZYK ${ }^{1}$, and ADAM FRONCZAK ${ }^{4}$}

\author{
${ }^{1}$ Medical University of Łódź, Łódź, Poland \\ Department of Preventive Medicine \\ ${ }^{2}$ Nofer Institute of Occupational Medicine, Łódź, Poland \\ Department of Work Physiology and Ergonomics \\ ${ }^{3}$ Medical University of Łódź, Łódź, Poland \\ Public Health Faculty \\ ${ }^{4}$ Ministry of Health, Warszawa, Poland
}

\begin{abstract}
Objective: The aim of current analysis was to identify socio-demographic correlates of heavy smoking. Materials and Methods: Global Adult Tobacco Survey (GATS), a nationally representative household study was implemented in Poland between 2009 and 2010. We used data on representative sample of 1915 adults, age 25 years and older. The Odds Ratios (OR) and 95\% Confidence Intervals (CI) for heavy smoking to the broad number of variables including age, age at smoking onset, education, occupational classification, living conditions, place of residence, place of residence at age about 14 years, awareness of smoking health consequences were tested in logistic regression model. Results: Among daily smokers the rate of heavy smokers was $63 \%$ in males and $45 \%$ in females $(\mathrm{p}<0.001)$. The present study indicated that three characteristics: age, early age at smoking onset and lack of awareness of smoking health consequences were significantly associated with heavy smoking among both genders. Significantly higher risk of heavy smoking was observed among the 50-59 years of age population compared to the youngest group. The heavy smoking rate was highest among males who started smoking under age 14 years $(\mathrm{OR}=3.1 ; 95 \% \mathrm{CI}: 1.4-6.7 ; \mathrm{p}<0.001)$ and females that started smoking at age $14-17$ years $(\mathrm{OR}=2.3 ; 95 \% \mathrm{CI}: 1.5-3.5 ; \mathrm{p}<0.0001)$ compared to those who started smoking at age 21 years or older. Heavy smoking was significantly correlated to lack of awareness of adverse health consequences of smoking $(\mathrm{OR}=1.5 ; 95 \% \mathrm{CI}: 1.1-2.03 ; \mathrm{p}<0.01$ and $\mathrm{OR}=1.5 ; 95 \% \mathrm{CI}: 1.0-2.2 ; \mathrm{p}<0.01$ for men and women, respectively) compared to aware respondents. Conclusions: These outcomes should be taken into account while developing tobacco control measures. Among other things, educational interventions to increase knowledge of adverse tobacco health effects should be widely implemented.
\end{abstract}

Key words:

Smoking, Tobacco control, Adults, Poland

Received: October 11, 2011. Accepted: November 22, 2011.

Address reprint request to D. Kaleta, Department of Preventive Medicine, Medical University of Łódź, Żeligowskiego 7/9, 90-752 Łódź, Poland (e-mail: dkaleta@op.pl). 


\section{INTRODUCTION}

Tobacco smoking is a leading, single, preventable cause of death among the adults. According to the World Health Organization (WHO), tobacco is the only legal consumer product that can harm everyone exposed to it and it kills up to half of those who use it [1]. Almost 6 million people die worldwide from tobacco use each year, both from direct use and environmental tobacco smoke [2]. It is estimated that by 2020, this number will increase to 7.5 million, accounting for $10 \%$ of all deaths. Smoking causes about $71 \%$ of lung cancer, $42 \%$ of chronic respiratory disease and close to $10 \%$ of cardiovascular diseases. In Poland, in 2000, tobacco smoking caused approximately 69000 deaths, of which about 43000 (37000 among men and 6000 among women) were premature deaths at the age of 35-69 years [3].

Numerous studies have demonstrated that health risk increases with increasing duration of exposure to the number of cigarettes smoked, and cumulative smoking dose [4-6]. It should be emphasized that heavy smokers, not only live on the average 10 years shorter than never smokers, but their health-related quality of life is significantly lower [7]. Limiting tobacco consumption may reduce health risk, while complete cessation of smoking is most effective. However, several studies have shown that heavy smokers are more nicotine-dependent, report greater difficulty quitting, are more troubled by withdrawal symptoms, experience stronger urges and cravings compared to light smokers $[8,9]$. These findings suggest that smoking cessation programs developed for heavy smokers may need to include strategies, like pharmacological treatment, enabling them to cope with the effects of the physical dependence [8]. Expanding the information on heavy smokers' characteristics is of key importance to both physicians and policy makers for developing and implementing more effective smoking policies and interventions. Although the serious health risks of heavy smoking are well known, data on smoking intensity, heavy smoking and its predictors are still poorly reported in the literature [10].

We evaluated socio-demographic correlates of heavy smoking among adult males and females in Poland.

\section{MATERIALS AND METHODS}

\section{Study design and sample}

Global Adult Tobacco Survey (GATS) was implemented in Poland under the Biennium Collaboration Agreement between the World Health Organization and the Ministry of Health. The Ministry of Health revised and approved all aspects of the project and supervised the whole process of the GATS implementation.

GATS is the global project developed for systematically monitoring adult tobacco use worldwide. GATS Poland is a standardized, household, nationally representative survey. GATS was implemented across 14 countries including China, Bangladesh, Brazil, Egypt, India, Thailand, Vietnam, Philippines, Mexico, Uruguay, Turkey, the Russian Federation, and Ukraine [11-13].

In Poland, the survey population selection process was based on multi-stage stratified geographically clustered sample of non-institutionalized population aged 15 years and older, including men and women. Subjects who lived in a school or students hostels, prisons, nursing homes and other institutions were not included. Data set was provided by the Central Statistical Office. A sample of 14000 households was randomly selected. Out of the 14000 households selected for the survey, 8948 (63.9\%) households and 7840 $(93.9 \%)$ sampled persons successfully completed the interviews. The total survey response rate was $65.1 \%$.

\section{Questionnaire design}

GATS questionnaire is composed of two parts: household and individual questionnaires. Household questionnaires contained general characteristics of the household 
members, which provided information that were used to determine who was eligible to participate in the survey. The individual GATS questionnaire has been divided into nine sections that cover the characteristics of the study participants and a number of important aspects of tobacco use including active and passive smoking, smokeless tobacco use, cessation of smoking.

In Poland, the modified version of GATS questionnaire was used to match the country-specific context. The field work was preceded by a number of trainings for all survey staff and a pretest. After the pretest that had been held in February 2009, minor changes were made to the GATS questionnaire, as well as some improvements in technical aspects of the survey and the operation of IT equipment. The GATS fieldwork was launched between November 2009 and March 2010. GATS data were collected by professional interviewers trained in the application and completion of the questionnaire. Questionnaires were administered at respondents' homes during face-to-face interviews. Fieldwork, data aggregation and all works during the study implementation process were carefully supervised.

\section{Study variables}

Current daily smoker was defined as a person who smokes regularly, at least 1 cigarette a day. Among daily smokers, we distinguished light to moderate smokers (smoking on average less than 20 cigarettes per day) and heavy smokers (smoking on average of 20 or more cigarettes per day).

Data on age at smoking onset were also included in our analysis in addition to gender and age of the respondents. Age at smoking onset was described as the age at which respondents started smoking on a regular basis. Based on the data obtained from the question "How old were you when you started smoking tobacco every day?", we emerged with four age groups of smoking initiation ( $<14,14-17,18-20,21$ years or over). We also used in our analysis the data on place of residence when respondent was about 14 years old, including village, small town (up to 50000 ), medium size town (50 000 to 100000 ), city over 100000 inhabitants.

Educational level was categorized as: primary education, vocational education, secondary education, and high education. The measure of economic activity classified subjects currently with permanent job as employed, currently with no permanent job as unemployed, and pupils, students, persons occupied with household keeping, retired, pensioners due to disability as economically non-active. Furthermore, subjects were asked whether their place of residence was a rural or urban area (urban area up to 50000 , from 50000 to 200000 , or over 200000 inhabitants). We also took into consideration the awareness of the health consequences of smoking. We categorized our respondents as aware (those who answered "yes" to the question: Do you think that tobacco smoking causes serious diseases?) and not aware (those who answered "no" and "do not know"). Moreover, we analyzed data on cohabitation of smokers (living alone, living with nonsmoker, living with smoker). Data on time to first cigarette after waking up (less than 30 minutes, 30 minutes and over), waking up during the night to smoke (yes, no), attempts to quit during past 12 months (yes, no) were also obtained.

\section{Statistical analyses}

Statistical associations of the given categories of characteristics in the analyzed groups were assessed with the chisquare test. Logistic regression model was implemented to identify factors associated with heavy smoking among males and females in the following age groups: 25-29, 30-39, 40-49, 50-59, 60 years and older. First, univariate coefficients - odds ratios (OR) of the impact of odd variables on the heavy smoking were calculated. Next, multifactorial analysis of the simultaneous effect of all variables on the risk of heavy smoking was applied. For all analyses, $p$ values less than 0.05 were set as statistically significant. The evaluation was completed using statistical software package STATISTICA Windows XP version 8.0. 


\section{RESULTS}

The prevalence of daily cigarette smoking was higher in males than females ( $31 \%$ vs. $22 \%$, p < 0.01). The mean age of smoking onset was 18.4 years $(\mathrm{SD}=3.62)$ in men compared to $20.1(\mathrm{SD}=4.81)$ years in women $(\mathrm{p}<0.001)$. Of the 3867 male respondents, $3 \%(\mathrm{n}=116)$ were occasional tobacco smokers, $28.7 \%(\mathrm{n}=1108)$ were former tobacco smokers, and $34.5 \%(\mathrm{n}=1334)$ were never smokers. Among the 3973 female respondents, the occasional, former, and never smokers were: $3.1 \%(\mathrm{n}=121) ; 16.1 \%$ $(\mathrm{n}=644)$; and 58.9\% $(\mathrm{n}=2338)$, respectively.

In the group of daily smokers, only four men and one woman smoked daily tobacco products other than commercially available and/or hand-rolled cigarettes. We excluded from the logistic regression analysis subjects younger than 25 years $($ men $n=32$; women $n=13$ ) and all records with missing data $(\mathrm{n}=219)$.

Finally, the data analyzed below embraced 1915 respondents, including 781 women and 1134 men aged 25 years or older who declared cigarette smoking on a daily basis.
The rate of heavy smokers among all respondents was significantly higher among males compared to females ( $20.5 \%$ vs. $9.5 \% \mathrm{p}<0.001)$. Among daily smokers, the rate of heavy smokers was $63 \%$ in males and $45 \%$ in females $(\mathrm{p}<0.001)$.

In the group of heavy smokers the mean age of smoking onset was 18.0 years $(\mathrm{SD}=3.46)$ in men vs. 19.4 years $(\mathrm{SD}=4.08)$ in women $(\mathrm{p}<0.001)$. Among light/moderate level smokers the mean age of smoking onset was 18.9 years $(\mathrm{SD}=3.77)$ in men vs. 20.5 years $(\mathrm{SD}=4.97)$ in women $(\mathrm{p}<0.001)$.

The mean number of cigarettes smoked daily in the group of male and female daily smokers was $18.6(\mathrm{SD}=8.88)$ vs. 15.7 ( $\mathrm{SD}=7.37$ ), respectively. The light to moderate smokers consumed an average of 10.9 (SD $=3.83$; ranging from 1 to $19 \mathrm{Me}=10$ ) cigarettes per day. The heavy smokers consumed an average of $23.1(\mathrm{SD}=7.09$; ranging from 20 to $130 \mathrm{Me}=20$ ) cigarettes per day.

Table 1 provides characteristics of light/moderate and heavy smokers by gender.

Table 1. Characteristics of light/moderate (less than 20 cigarettes per day) and heavy (20 and more cigarettes per day) smokers aged 25 years and older by gender - Global Adult Tobacco Survey Poland 2009-2010

\begin{tabular}{|c|c|c|c|c|c|c|}
\hline \multirow[b]{2}{*}{ Characteristic } & \multicolumn{3}{|c|}{$\begin{array}{l}\text { Male } \\
\mathrm{n}(\%)\end{array}$} & \multicolumn{3}{|c|}{$\begin{array}{c}\text { Female } \\
\mathrm{n}(\%)\end{array}$} \\
\hline & $\begin{array}{c}\text { total } \\
(\mathrm{N}=1134)\end{array}$ & $\begin{array}{c}\text { light/ } \\
\text { moderate } \\
\text { smokers } \\
\text { (less than } \\
20 \text { cigarettes } \\
\text { per day) }\end{array}$ & $\begin{array}{l}\text { heavy smokers } \\
\text { (20 and more } \\
\text { cigarettes } \\
\text { per day) }\end{array}$ & $\begin{array}{c}\text { total } \\
(\mathrm{N}=781)\end{array}$ & $\begin{array}{c}\text { light/ } \\
\text { moderate } \\
\text { smokers } \\
\text { (less than } \\
20 \text { cigarettes } \\
\text { per day) }\end{array}$ & $\begin{array}{c}\text { heavy smokers } \\
\text { (20 and more } \\
\text { cigarettes } \\
\text { per day) }\end{array}$ \\
\hline \multicolumn{7}{|l|}{ Age (years) } \\
\hline $25-29$ & $133(11.7)$ & $79(18.8)$ & $54(7.6)^{\mathrm{a}}$ & $86(11.0)$ & $58(13.5)$ & $28(8.0)^{\mathrm{c}}$ \\
\hline $30-39$ & $279(24.6)$ & $96(22.8)$ & $183(25.7)$ & $159(20.4)$ & $98(22.8)$ & $61(17.4)$ \\
\hline $40-49$ & $297(26.2)$ & $101(24.0)$ & $196(27.5)$ & $208(26.6)$ & $108(25.1)$ & $100(28.5)$ \\
\hline $50-59$ & $265(23.4)$ & $84(20.0)$ & $181(25.3)^{\mathrm{c}}$ & $238(30.5)$ & $114(26.5)$ & $124(35.3)^{\mathrm{b}}$ \\
\hline$\geq 60$ & $160(14.1)$ & $61(14.4)$ & $99(13.9)$ & $90(11.5)$ & $52(12.1)$ & $38(10.8)$ \\
\hline \multicolumn{7}{|l|}{ Age at smoking onset } \\
\hline$<14$ & $39(3.4)$ & $10(2.4)$ & $29(4.1)$ & $5(0.6)$ & $3(0.7)$ & $2(0.6)$ \\
\hline
\end{tabular}


Table 1. Characteristics of light/moderate (less than 20 cigarettes per day) and heavy (20 and more cigarettes per day) smokers aged 25 years and older by gender - Global Adult Tobacco Survey Poland 2009-2010 - cont.

\begin{tabular}{|c|c|c|c|c|c|c|}
\hline \multirow[b]{2}{*}{ Characteristic } & \multicolumn{3}{|c|}{$\begin{array}{l}\text { Male } \\
\mathrm{n}(\%)\end{array}$} & \multicolumn{3}{|c|}{$\begin{array}{c}\text { Female } \\
\mathrm{n}(\%)\end{array}$} \\
\hline & $\begin{array}{c}\text { total } \\
(\mathrm{N}=1134)\end{array}$ & $\begin{array}{c}\text { light/ } \\
\text { moderate } \\
\text { smokers } \\
\text { (less than } \\
20 \text { cigarettes } \\
\text { per day) }\end{array}$ & $\begin{array}{l}\text { heavy smokers } \\
\text { (20 and more } \\
\text { cigarettes } \\
\text { per day) }\end{array}$ & $\begin{array}{c}\text { total } \\
(\mathrm{N}=781)\end{array}$ & $\begin{array}{c}\text { light/ } \\
\text { moderate } \\
\text { smokers } \\
\text { (less than } \\
20 \text { cigarettes } \\
\text { per day) }\end{array}$ & $\begin{array}{l}\text { heavy smokers } \\
\text { (20 and more } \\
\text { cigarettes } \\
\text { per day) }\end{array}$ \\
\hline $14-17$ & $402(35.4)$ & $129(30.6)$ & $273(38.2)^{\mathrm{b}}$ & $197(25.3)$ & $91(21.2)$ & $106(30.2)^{b}$ \\
\hline $18-20$ & $474(41.8)$ & $169(40.2)$ & $305(42.8)$ & $349(44.7)$ & $194(45.1)$ & $155(44.2)$ \\
\hline$\geq 21$ & $219(19.4)$ & $113(26.8)$ & $106(14.9)^{\mathrm{a}}$ & $230(29.4)$ & $142(33.0)$ & $88(25.1)^{\mathrm{c}}$ \\
\hline \multicolumn{7}{|l|}{ Education } \\
\hline primary & $184(16.2)$ & $71(16.8)$ & $113(15.8)$ & $107(13.7)$ & $58(13.5)$ & $49(14.0)$ \\
\hline vocational & $505(44.5)$ & $164(39.0)$ & $341(47.8)^{b}$ & $228(29.2)$ & $120(27.9)$ & $108(30.8)$ \\
\hline secondary & $356(31.5)$ & $148(35.1)$ & $208(29.2)^{c}$ & $335(42.9)$ & $184(42.7)$ & $151(43.0)$ \\
\hline high & $89(7.8)$ & $38(9.1)$ & $51(7.2)$ & $111(14.2)$ & $68(15.9)$ & $43(12.2)$ \\
\hline \multicolumn{7}{|l|}{ Occupational classification } \\
\hline non-economically active & $272(24.0)$ & $99(23.6)$ & $173(24.3)$ & $303(38.8)$ & $164(38.1)$ & $139(39.6)$ \\
\hline employed & $743(65.5)$ & $281(66.7)$ & $462(64.8)$ & $428(54.8)$ & $242(56.3)$ & $186(53.0)$ \\
\hline $\begin{array}{l}\text { unemployed, currently with } \\
\text { no permanent job }\end{array}$ & $119(10.5)$ & $41(9.7)$ & $78(10.9)$ & $50(6.4)$ & $24(5.6)$ & $26(7.4)$ \\
\hline \multicolumn{7}{|l|}{ Living conditions } \\
\hline living with nonsmoker & $94(8.3)$ & $31(7.4)$ & $63(8.8)$ & $65(8.3)$ & $30(7.0)$ & $35(10.0)$ \\
\hline living with smoker & $168(14.8)$ & $69(16.4)$ & $99(13.9)^{\mathrm{b}}$ & $109(14.0)$ & $59(13.7)$ & $50(14.2)$ \\
\hline living alone & $872(76.9)$ & $321(76.2)$ & $551(77.3)$ & $607(77.7)$ & $341(79.3)$ & $266(75.8)$ \\
\hline \multicolumn{7}{|l|}{ Place of residence } \\
\hline $\begin{array}{l}\text { rural } \\
\text { urban }\end{array}$ & $598(52.7)$ & $212(50.4)$ & $386(54.1)$ & $306(39.2)$ & $164(38.1)$ & $142(40.5)$ \\
\hline up to 50000 & $200(17.6)$ & $85(20.2)$ & $115(16.1)$ & $143(18.3)$ & $82(19.1)$ & $61(17.4)$ \\
\hline $50000-200000$ & $147(13.0)$ & $39(9.2)$ & $108(15.2)^{\mathrm{b}}$ & $138(17.7)$ & $86(20.0)$ & $52(14.8)$ \\
\hline over 200000 & $189(16.7)$ & $85(20.2)$ & $104(14.6)^{c}$ & $194(24.8)$ & $98(22.8)$ & $96(27.4)$ \\
\hline \multicolumn{7}{|l|}{$\begin{array}{l}\text { Place of residence when were } \\
\text { about } 14 \text { years old }\end{array}$} \\
\hline $\begin{array}{l}\text { village } \\
\text { urban }\end{array}$ & $595(52.5)$ & $206(48.9)$ & $389(54.6)$ & $342(43.8)$ & $191(44.4)$ & $151(43.0)$ \\
\hline small town up to 50000 & $229(20.2)$ & $100(23.8)$ & $129(18.1)^{c}$ & $151(19.3)$ & $84(19.5)$ & $67(19.1)$ \\
\hline $\begin{array}{l}\text { medium size } \\
\text { town } 50000-100000\end{array}$ & $114(10.1)$ & $35(8.3)$ & 79 (11.1) & $110(14.1)$ & $58(13.5)$ & $52(14.8)$ \\
\hline city over 100000 & $196(17.2)$ & $80(19.0)$ & $116(16.2)$ & $178(22.8)$ & $97(22.6)$ & $81(23.1)$ \\
\hline
\end{tabular}


Table 1. Characteristics of light/moderate (less than 20 cigarettes per day) and heavy (20 and more cigarettes per day) smokers aged 25 years and older by gender - Global Adult Tobacco Survey Poland 2009-2010 - cont.

\begin{tabular}{|c|c|c|c|c|c|c|}
\hline \multirow[b]{2}{*}{ Characteristic } & \multicolumn{3}{|c|}{$\begin{array}{l}\text { Male } \\
\mathrm{n}(\%)\end{array}$} & \multicolumn{3}{|c|}{$\begin{array}{c}\text { Female } \\
\mathrm{n}(\%)\end{array}$} \\
\hline & $\begin{array}{c}\text { total } \\
(\mathrm{N}=1134)\end{array}$ & $\begin{array}{c}\text { light/ } \\
\text { moderate } \\
\text { smokers } \\
\text { (less than } \\
20 \text { cigarettes } \\
\text { per day) }\end{array}$ & $\begin{array}{l}\text { heavy smokers } \\
\text { (20 and more } \\
\text { cigarettes } \\
\text { per day) }\end{array}$ & $\begin{array}{c}\text { total } \\
(\mathrm{N}=781)\end{array}$ & $\begin{array}{c}\text { light/ } \\
\text { moderate } \\
\text { smokers } \\
\text { (less than } \\
20 \text { cigarettes } \\
\text { per day) }\end{array}$ & $\begin{array}{l}\text { heavy smokers } \\
\text { (20 and more } \\
\text { cigarettes } \\
\text { per day) }\end{array}$ \\
\hline \multicolumn{7}{|c|}{$\begin{array}{l}\text { Awareness of smoking health } \\
\text { consequences }\end{array}$} \\
\hline yes & $911(80.3)$ & $353(83.8)$ & $558(78.3)^{\mathrm{c}}$ & $642(82.2)$ & $367(85.3)$ & $275(78.3)^{c}$ \\
\hline no & $223(19.7)$ & $68(16.2)$ & $155(21.7)^{\mathrm{c}}$ & $139(17.8)$ & $63(14.7)$ & $76(21.7)^{c}$ \\
\hline \multicolumn{7}{|l|}{ Time to first cigarette } \\
\hline$<30 \min$ & $732(64.8)$ & $178(42.4)$ & $554(78.1)^{\mathrm{a}}$ & $454(58.3)$ & $176(41.0)$ & $278(79.4)^{\mathrm{a}}$ \\
\hline$\geq 30 \mathrm{~min}$ & $397(35.2)$ & $242(57.6)$ & $155(21.9)^{\mathrm{a}}$ & $325(41.7)$ & $253(59.0)$ & $72(20.6)^{\mathrm{a}}$ \\
\hline \multicolumn{7}{|c|}{ Wake up at night to smoke } \\
\hline yes & $283(25.0)$ & $51(12.1)$ & $232(32.6)^{\mathrm{a}}$ & $170(21.8)$ & $43(10.0)$ & $127(36.3)^{\mathrm{a}}$ \\
\hline no & $848(75.0)$ & $369(87.9)$ & $479(67.4)^{\mathrm{a}}$ & $609(78.2)$ & $386(90.0)$ & $223(63.7)^{\mathrm{a}}$ \\
\hline \multicolumn{7}{|l|}{$\begin{array}{l}\text { Quit attempts during } \\
\text { the past } 12 \text { months }\end{array}$} \\
\hline yes & $328(28.9)$ & $137(32.5)$ & $191(26.8)^{c}$ & $241(30.9)$ & $153(35.6)$ & $88(25.1)^{\mathrm{b}}$ \\
\hline no & $806(71.1)$ & $284(67.5)$ & $522(73.2)^{\mathrm{c}}$ & $540(69.1)$ & $277(64.4)$ & $263(74.9)^{\mathrm{b}}$ \\
\hline
\end{tabular}

${ }^{a}$ Light/moderate smokers vs. heavy smokers $\mathrm{p} \leq 0.001$.

${ }^{\mathrm{b}}$ Light/moderate smokers vs. heavy smokers $\mathrm{p} \leq 0.01$.

${ }^{\mathrm{c}}$ Light/moderate smokers vs. heavy smokers $\mathrm{p} \leq 0.05$.

In the female population, higher rate of heavy smokers compared to light/moderate smokers was observed among subjects aged 25-29 and 50-59 years and among those who started smoking on regular basis at the age of 14-17 years. A higher number of heavy smokers compared to those smoking less than 20 cigarettes per day was found in the groups: not aware of smoking health consequences, those who smoke their first cigarette within less than 30 minutes after waking up, waking up at night to smoke, and among subjects who did not declare any attempt to quit smoking during the past 12 months. Among men, we established higher prevalence of heavy smokers compared to lighter smokers in the groups; 50-59 years, age at smoking onset 14-17 years, men with vocational education, living in a medium size urban area. Similarly among women, a higher number of smokers was found among respondents that were not aware of the adverse health effects of smoking, smoking their first cigarette within less than 30 minutes after waking up, waking up at night to smoke, and subjects who did not declare any attempt to quit smoking during the past year.

Additionally, Odds Ratios (OR) and 95\% Confidence Intervals (CI) for heavy smoking to the broad number of variables including age, age at smoking onset, education, occupational classification, living conditions, place of residence, place of residence at age about 14 years, awareness 
of smoking health consequences, was tested in a logistic regression model.

Three characteristics: age, age at smoking onset and awareness of smoking health consequences were significantly associated with heavy smoking among both genders. Results of the univariate and multivariate analysis are displayed in Tables 2 and 3 .

\section{Univariate analysis}

The heavy smoking rate was the highest among the male subjects of 50 to 59 years of age compared to those aged 25-29 years $(\mathrm{OR}=3.1 ; 95 \% \mathrm{CI}: 2.0-4.9 ; \mathrm{p}<0.0001)$. The highest percentages of heavy smokers were observed among males that started smoking on regular basis before age $14(\mathrm{OR}=3.1 ; 95 \% \mathrm{CI}$ : $1.4-6.7)$ compared to

Table 2. Odds Ratios (OR) and 95\% Confidence Intervals (CI) for heavy smoking to selected socio-demographic characteristics in men at age 25 and over $(n=1134)$ - Global Adult Tobacco Survey Poland (2009-2010)

\begin{tabular}{|c|c|c|c|c|c|c|c|}
\hline \multirow{2}{*}{ Variable } & \multirow{2}{*}{$\begin{array}{c}\text { Total } \\
\text { (n) }\end{array}$} & \multicolumn{2}{|c|}{ Heavy smokers } & \multicolumn{2}{|c|}{$\begin{array}{l}\text { Univariate logistic } \\
\text { regression }\end{array}$} & \multicolumn{2}{|c|}{$\begin{array}{l}\text { Multivariate logistic } \\
\text { regression }^{\mathrm{a}}\end{array}$} \\
\hline & & $\mathrm{n}$ & $\%$ & OR & $95 \% \mathrm{CI}$ & OR & $95 \% \mathrm{CI}$ \\
\hline \multicolumn{8}{|l|}{ Age (years) } \\
\hline $25-29$ & 133 & 54 & 40.6 & 1.00 & reference & 1.00 & reference \\
\hline $30-39$ & 279 & 183 & 65.6 & $2.79^{\mathrm{b}}$ & $1.82-4.27$ & $3.06^{\mathrm{b}}$ & $1.95-4.78$ \\
\hline $40-49$ & 297 & 196 & 66.0 & $2.84^{\mathrm{b}}$ & $1.86-4.33$ & $3.03^{\mathrm{b}}$ & $1.93-4.78$ \\
\hline $50-59$ & 265 & 181 & 68.3 & $3.15^{\mathrm{b}}$ & $2.04-4.86$ & $3.52^{\mathrm{b}}$ & $2.18-5.67$ \\
\hline$\geq 60$ & 160 & 99 & 61.9 & $2.37^{\mathrm{b}}$ & $1.48-3.80$ & $2.97^{\mathrm{b}}$ & $1.60-5.53$ \\
\hline \multicolumn{8}{|l|}{ Age at smoking onset } \\
\hline$<14$ & 39 & 29 & 74.4 & $3.09^{c}$ & $1.44-6.66^{c}$ & $3.91^{\mathrm{b}}$ & $1.74-8.80$ \\
\hline $14-17$ & 402 & 273 & 67.9 & $2.26^{\mathrm{b}}$ & $1.61-3.16$ & $2.55^{\mathrm{b}}$ & $1.78-3.67$ \\
\hline $18-20$ & 474 & 305 & 64.3 & $1.92^{\mathrm{b}}$ & $1.39-2.66$ & $2.08^{\mathrm{b}}$ & $1.48-2.92$ \\
\hline$\geq 21$ & 219 & 106 & 48.4 & 1.00 & reference & 1.00 & reference \\
\hline \multicolumn{8}{|l|}{ Education } \\
\hline primary & 184 & 113 & 61.4 & 1.19 & $0.71-1.98$ & 0.73 & $0.40-1.33$ \\
\hline vocational & 505 & 341 & 67.5 & 1.55 & $0.98-2.45$ & 1.05 & $0.63-1.75$ \\
\hline secondary & 356 & 208 & 58.4 & 1.05 & $0.65-1.66$ & 0.86 & $0.52-1.43$ \\
\hline high & 89 & 51 & 57.3 & 1.00 & reference & 1.00 & reference \\
\hline \multicolumn{8}{|l|}{ Occupational classification } \\
\hline economically not active & 272 & 173 & 63.6 & 1.00 & reference & 1.00 & reference \\
\hline employed & 743 & 462 & 62.2 & 0.94 & $0.71-1.26$ & 1.08 & $0.72-1.62$ \\
\hline $\begin{array}{l}\text { unemployed-currently with } \\
\text { no permanent job }\end{array}$ & 119 & 78 & 65.5 & 1.09 & $0.69-1.71$ & 1.18 & $0.69-2.03$ \\
\hline \multicolumn{8}{|l|}{ Living conditions } \\
\hline living with nonsmoker & 94 & 63 & 67.0 & 1.00 & reference & 1.00 & reference \\
\hline living with smoker & 168 & 99 & 58.9 & 0.71 & $0.42-1.20$ & 0.70 & $0.40-1.23$ \\
\hline living alone & 872 & 551 & 63.2 & 0.84 & $0.54-1.33$ & 0.87 & $0.54-1.40$ \\
\hline \multicolumn{8}{|l|}{ Place of residence } \\
\hline rural & 598 & 386 & 64.5 & 1.00 & reference & 1.00 & reference \\
\hline
\end{tabular}


Table 2. Odds Ratios (OR) and 95\% Confidence Intervals (CI) for heavy smoking to selected socio-demographic characteristics in men at age 25 and over $(n=1134)$ - Global Adult Tobacco Survey Poland (2009-2010) - cont.

\begin{tabular}{|c|c|c|c|c|c|c|c|}
\hline \multirow{2}{*}{ Variable } & \multirow{2}{*}{$\begin{array}{c}\text { Total } \\
\text { (n) }\end{array}$} & \multicolumn{2}{|c|}{ Heavy smokers } & \multicolumn{2}{|c|}{$\begin{array}{l}\text { Univariate logistic } \\
\text { regression }\end{array}$} & \multicolumn{2}{|c|}{$\begin{array}{l}\text { Multivariate logistic } \\
\text { regression }^{\mathrm{a}}\end{array}$} \\
\hline & & $\mathrm{n}$ & $\%$ & OR & $95 \% \mathrm{CI}$ & OR & $95 \% \mathrm{CI}$ \\
\hline \multicolumn{8}{|l|}{ urban } \\
\hline$<50000$ & 200 & 115 & 57.5 & 0.74 & $0.54-1.03$ & 0.80 & $0.51-1.25$ \\
\hline $50000-200000$ & 147 & 108 & 73.5 & 1.52 & $0.98-1.78$ & 1.44 & $0.83-2.49$ \\
\hline$>200000$ & 189 & 104 & 55.0 & 0.67 & $0.51-1.08$ & 0.75 & $0.46-1.23$ \\
\hline \multicolumn{8}{|l|}{$\begin{array}{l}\text { Place of residence when } \\
\text { about } 14 \text { years old }\end{array}$} \\
\hline $\begin{array}{l}\text { village } \\
\text { urban }\end{array}$ & 595 & 389 & 65.4 & 1.00 & reference & 1.00 & reference \\
\hline small town $<50000$ & 229 & 129 & 56.3 & 0.68 & $0.59-1.03$ & 0.72 & $0.47-1.10$ \\
\hline $\begin{array}{l}\text { medium size } \\
\text { town } 50000-100000\end{array}$ & 114 & 79 & 69.3 & 1.20 & $0.93-1.63$ & 1.15 & $0.64-2.03$ \\
\hline city > 100000 & 196 & 116 & 59.2 & 0.77 & $0.55-1.07$ & 0.87 & $0.52-1.45$ \\
\hline \multicolumn{8}{|c|}{$\begin{array}{l}\text { Awareness of smoking health } \\
\text { consequences }\end{array}$} \\
\hline yes & 911 & 558 & 61.3 & 1.00 & reference & 1.00 & reference \\
\hline no & 223 & 155 & 69.5 & $1.44^{\mathrm{d}}$ & $1.05-1.98$ & $1.46^{\mathrm{d}}$ & $1.05-2.03$ \\
\hline
\end{tabular}

${ }^{a}$ Fully adjusted model including: age, age at smoking onset, education, occupational classification, living conditions, place of residence, place of residence at age about 14 years, awareness of smoking health consequences.

${ }^{\mathrm{b}} \mathrm{p} \leq 0.001$.

${ }^{\mathrm{c}} \mathrm{p} \leq 0.01$.

${ }^{\mathrm{d}} \mathrm{p} \leq 0.05$.

Table 3. Odds Ratios (OR) and 95\% Confidence Intervals (CI) for heavy smoking to selected socio-demographic characteristics in women at age 25 and older ( $=781)$ - Global Adult Tobacco Survey Poland (2009-2010)

\begin{tabular}{|c|c|c|c|c|c|c|c|}
\hline \multirow[t]{2}{*}{ Variable } & \multirow{2}{*}{$\begin{array}{c}\text { Total } \\
\text { (n) }\end{array}$} & \multicolumn{2}{|c|}{ Heavy smokers } & \multicolumn{2}{|c|}{$\begin{array}{l}\text { Univariate logistic } \\
\text { regression }\end{array}$} & \multicolumn{2}{|c|}{$\begin{array}{l}\text { Multivariate logistic } \\
\text { regression }^{\mathrm{a}}\end{array}$} \\
\hline & & $\mathrm{n}$ & $\%$ & OR & $95 \% \mathrm{CI}$ & OR & $95 \% \mathrm{CI}$ \\
\hline \multicolumn{8}{|l|}{ Age (years) } \\
\hline 25-29 & 86 & 28 & 32.6 & 1.00 & reference & 1.00 & reference \\
\hline $30-39$ & 159 & 61 & 38.4 & 1.29 & $0.74-2.24$ & 1.41 & $0.79-2.51$ \\
\hline $40-49$ & 208 & 100 & 48.1 & $1.92^{\mathrm{b}}$ & $1.13-3.25$ & $2.30^{\mathrm{c}}$ & $1.30-4.06$ \\
\hline $50-59$ & 238 & 124 & 52.1 & $2.25^{\mathrm{c}}$ & $1.34-3.79$ & $2.75^{\mathrm{c}}$ & $1.57-4.80$ \\
\hline$\geq 60$ & 90 & 38 & 42.2 & 1.51 & $0.82-2.80$ & $2.08^{\mathrm{b}}$ & $1.05-4.15$ \\
\hline \multicolumn{8}{|l|}{ Age at smoking onset } \\
\hline$<14$ & 5 & 2 & 40.0 & 1.06 & $0.18-6.67$ & 1.46 & $0.23-9.27$ \\
\hline $14-17$ & 197 & 106 & 53.8 & $1.88^{\mathrm{c}}$ & $1.28-2.77$ & $2.26^{\mathrm{d}}$ & $1.49-3.45$ \\
\hline $18-20$ & 349 & 155 & 44.4 & 1.29 & $0.92-1.81$ & $1.46^{\mathrm{b}}$ & $1.02-2.09$ \\
\hline$\geq 21$ & 230 & 88 & 38.3 & 1.00 & reference & 1.00 & reference \\
\hline
\end{tabular}


Table 3. Odds Ratios (OR) and 95\% Confidence Intervals (CI) for heavy smoking to selected socio-demographic characteristics in women at age 25 and older ( $=781$ - Global Adult Tobacco Survey Poland (2009-2010) - cont.

\begin{tabular}{|c|c|c|c|c|c|c|c|}
\hline \multirow[t]{2}{*}{ Variable } & \multirow{2}{*}{$\begin{array}{c}\text { Total } \\
\text { (n) }\end{array}$} & \multicolumn{2}{|c|}{ Heavy smokers } & \multicolumn{2}{|c|}{$\begin{array}{l}\text { Univariate logistic } \\
\text { regression }\end{array}$} & \multicolumn{2}{|c|}{$\begin{array}{l}\text { Multivariate logistic } \\
\text { regression }^{\mathrm{a}}\end{array}$} \\
\hline & & $\mathrm{n}$ & $\%$ & OR & $95 \% \mathrm{CI}$ & OR & $95 \% \mathrm{CI}$ \\
\hline \multicolumn{8}{|l|}{ Education } \\
\hline primary & 107 & 49 & 45.8 & 1.34 & $0.78-2.29$ & 0.98 & $0.54-1.79$ \\
\hline vocational & 228 & 108 & 47.4 & 1.42 & $0.90-2.26$ & 1.09 & $0.65-1.82$ \\
\hline secondary & 335 & 151 & 45.1 & 1.30 & $0.84-2.01$ & 1.09 & $0.69-1.74$ \\
\hline high & 111 & 43 & 38.7 & 1.00 & reference & 1.00 & reference \\
\hline \multicolumn{8}{|l|}{ Occupational classification } \\
\hline economically not active & 303 & 139 & 45.9 & 1.00 & reference & 1.00 & reference \\
\hline employed & 428 & 186 & 43.5 & 0.91 & $0.67-1.22$ & 0.88 & $0.62-1.27$ \\
\hline $\begin{array}{l}\text { unemployed-currently with } \\
\text { no permanent job }\end{array}$ & 50 & 26 & 52.0 & 1.28 & $0.70-2.32$ & 1.27 & $0.67-2.41$ \\
\hline \multicolumn{8}{|l|}{ Living conditions } \\
\hline living with nonsmoker & 65 & 35 & 53.8 & 1.00 & reference & 1.00 & reference \\
\hline living with smoker & 109 & 50 & 45.9 & 0.73 & $0.39-1.35$ & 0.88 & $0.46-1.69$ \\
\hline living alone & 607 & 266 & 43.8 & 0.67 & $0.40-1.12$ & 0.77 & $0.45-1.69$ \\
\hline \multicolumn{8}{|l|}{ Place of residence } \\
\hline rural & 306 & 142 & 46.4 & 1.00 & reference & 1.00 & reference \\
\hline \multicolumn{8}{|l|}{ urban } \\
\hline$>50000$ & 143 & 61 & 42.7 & 0.86 & $0.57-1.28$ & 0.70 & $0.43-1.13$ \\
\hline $50000-200000$ & 138 & 52 & 37.7 & 0.70 & $0.46-1.05$ & $0.54^{\mathrm{b}}$ & $0.32-0.91$ \\
\hline$<200000$ & 194 & 96 & 49.5 & 1.13 & $0.79-1.62$ & 1.19 & $0.73-1.94$ \\
\hline \multicolumn{8}{|l|}{$\begin{array}{l}\text { Place of residence when } \\
\text { about } 14 \text { years old }\end{array}$} \\
\hline village & 342 & 151 & 44.2 & 1.00 & reference & 1.00 & reference \\
\hline \multicolumn{8}{|l|}{ urban } \\
\hline small town $<50000$ & 151 & 67 & 44.4 & 1.01 & $0.68-1.49$ & 1.10 & $0.70-1.73$ \\
\hline $\begin{array}{l}\text { medium size town } \\
50000-100000\end{array}$ & 110 & 52 & 47.3 & 1.13 & $0.74-1.75$ & 1.60 & $0.93-2.75$ \\
\hline city $>100000$ & 178 & 81 & 45.5 & 1.61 & $0.73-1.52$ & 1.06 & $0.64-1.77$ \\
\hline \multicolumn{8}{|l|}{$\begin{array}{l}\text { Awareness of smoking health } \\
\text { consequences }\end{array}$} \\
\hline yes & 642 & 275 & 42.8 & 1.00 & reference & 1.00 & reference \\
\hline no & 139 & 76 & 54.7 & $1.6^{\mathrm{b}}$ & $1.11-2.33$ & $1.53^{\mathrm{b}}$ & $1.04-2.24$ \\
\hline
\end{tabular}

${ }^{a}$ Fully adjusted model including: age, age at smoking onset, education, occupational classification, living conditions, place of residence, place of residence at age about 14 years, awareness of smoking health consequences.

${ }^{\mathrm{b}} \mathrm{p} \leq 0.05$.

${ }^{\mathrm{c}} \mathrm{p} \leq 0.01$.

${ }^{\mathrm{d}} \mathrm{p} \leq 0.001$. 
those who started smoking at the age of 21 years or later. Among the male population, the risk of heavy smoking was 1.5 times higher in the group that did not perceive smoking as dangerous to health, compared to the people that considered smoking to cause serious illnesses (OR $=1.4 ; 95 \%$ CI: 1.0-2.0; $\mathrm{p}<0.01)$.

Similarly, among women, heavy smoking rate was higher among those aged 50-59 than among youngest group 20-29 years $(\mathrm{OR}=2.5 ; 95 \% \mathrm{CI} 1.3-3.8 ; \mathrm{p}<0.001)$. In women, age under 14 at smoking onset was a significant determinant of heavy smoking. Furthermore, in women, lack of awareness of health risks of smoking was also a significant determinant of heavy smoking (OR = 1.6; 95\% CI: 1.1-2.3; $\mathrm{p}<0.01)$.

\section{Multivariate analysis}

The multivariate analysis confirmed the results observed in the univariate section (Tables 3 and 4). The significantly higher risk of heavy smoking was observed among the males 50-59 years of age compared to the youngest population (OR $=3.5 ; 95 \%$ CI: 2.2-5.7; $<<0.0001)$.

Heavy smoking was strongly associated with starting to smoke at a young age. Heavy smoking in men was significantly correlated to lack of awareness of adverse health consequences of smoking as well $(\mathrm{OR}=1.5 ; 95 \% \mathrm{CI}$ : 1.1-2.03; $\mathrm{p}<0.01)$.

For the women, heavy smoking was also associated with age and age of smoking onset. Similar to the male subjects, association between lack of awareness of health consequences of smoking and heavy smoking was observed for the female respondents $(\mathrm{OR}=1.5 ; 95 \% \mathrm{CI}$ : $1.0-2.2$; $\mathrm{p}<0.01)$.

\section{DISCUSSION}

In our study, as well as in other surveys, heavy smoking was significantly more prevalent in men than women $[9,14,15]$. However, we noticed significantly higher rates of heavy smoking subjects compared to surveys from United States. Of those who smoke in Poland, 63\% males and $45 \%$ females were heavy smokers, while in reports by Wilson et al. $35 \%$ of men and $24 \%$ of women were classified as heavy smokers $[9,15]$. This difference can be explained by the fact that data were collected in different years and different criteria were adopted to describe heavy smokers. The recent study by Baumert et al. shows similar results [14]. Moreover, our results are consistent with previous reports suggesting that, compared with lighter smokers, heavy-level smokers were less likely to represent younger age groups $[9,14]$. The highest rates of heavy smokers were mostly found in mid-age (30 years or older) subpopulations [9, 15]. Findings of Sorensen et al. suggest that, compared to lighter smokers, heavy smokers may depend more on nicotine and are likely to respond to a broader array of cues to smoke, which are factors that appear to contribute to heavy smokers' greater difficulties with quitting [16]. In our study, heavy smokers were also more likely to smoke their first cigarette of the day within 30 min of waking up, which suggests that they were more nicotine dependent. Major barriers to quitting for female heavy smokers include: a lack of confidence in their ability to quit, insufficient tools to succeed with cessation attempts, and fear that quitting may result in weight gain [16]. High prevalence of heavy smoking among older people is perhaps the result of difficulty in quitting smoking or ineffectiveness of anti-smoking campaigns. The majority of Polish smokers declare their will to quit smoking and many of them attempt to quit. Most smokers (77\%), however, attempted to quit on their own, which could not be effective, especially among those smoking a great number of cigarettes per day [17]. All forms of nicotine replacement therapy (NRT) can help increase chances to quit smoking successfully among people who make a quit attempt [18]. NRTs increase the rate of quitting by $50-70 \%$. Other types of medication were also found to increase effectiveness of therapy, but in Poland they 
are still quite expensive and not reimbursed by National Health Fund.

Smoking causes nicotine addiction over time which leads to increasing the number of cigarettes smoked [19]. This problem is particularly important in relation to persons who started smoking tobacco regularly at young age. Early age (under 14 years) at smoking onset is unquestionably the most important risk factor, with 3.9 (95\% CI: 1.7-8.8) multivariate odds of heavy smoking among men. Results from MONICA/KORA Augsburg Survey confirmed such correlation [14]. However, in contrast to their findings, we observed association between being heavy smokers and early age at smoking onset in females. It is difficult to definitely determine what factors were responsible for the different results of the German MONICA study. We may only speculate that the dissimilarity might be associated with differences among countries in comprehensiveness and effectiveness of implemented tobacco control measures, such as e.g., implementation of country-specific interventions and polices in Germany with special focus on young girls [20]. In contrast to other observations, we did not find a connection of heavy smoking with economic factors $[9,14,15]$. But subjects that were not aware of the health risks of smoking were more likely to be smokers compared to those that were aware. Some reports show that the knowledge on harmfulness of tobacco use is still insufficient in our country. Study by Sieminska et al. reported, for instance, that the most important reasons for quitting smoking among adults were: general health concern $(57 \%)$, personal health problems (32\%) and social reasons (32\%). However, $41 \%$ of smokers prompted to quitting by personal health problems related to tobacco smoking did not see the link between the two [17]. Health knowledge seems to be one of the most important factors limiting heavy smoking.

Education and information about the hazards of smoking should be improved. On the other hand, knowledge of benefits from quitting and reduction of health risk with smoking cessation should be expanded [20].

\section{Study limitations and strengths}

Most studies implement different criteria and methods to measure tobacco use [21-23]. As a potential limitation of GATS, the recall bias due to self-reported data on tobacco use should be mentioned.

There is always the possibility that data about smoking status obtained by questionnaire interview may be underestimated as the people might not say the truth about their smoking habit. Questionnaire surveys collect data on what people say, not necessarily what they do. So if general norms shift and people tend to give more acceptable answers, then a policy that influences general awareness might appear to have a stronger impact on smoking behaviour than indeed it has. There is a huge number of objective measures, markers or biomarkers of tobacco smoking including nicotine, cotinine, carbon monoxide that are used [24]. However, due to high costs and time-consuming procedures, such methods are not widely used for large population surveys. Moreover, the need of collecting saliva, urine or blood for biomarker analysis can increase the number of refusals and lead to non-participation bias. In this light, questionnaires seem to be relatively economical, easy to collect, allow approaching high number of subjects, and have been found to be valid measures in most epidemiological studies [25]. Cross sectional design of the study describes a single point in time, and it limits our ability to conclude on heavy smoking predictors during the life span, and determine whether the associations we found will change or remain. Another potential limitation of epidemiological studies is recall bias. However, response rate in GATS was over $60 \%$ of the typical level, or even higher than in other nationwide population-representative questionnaire surveys in Poland [26]. Moreover, GATS is a nationally representative household survey of adults 15 years of age or older using a standard core questionnaire, sample 
design, and data collection and management procedures that have been reviewed and approved by international experts.

To our knowledge, GATS has provided probably the best and the most recent data available on smoking in Poland. The data derived from GATS will strongly support policy makers in their future work aiming at tobacco control in Poland. Experience gained during the survey implementation will, in the future, allow building systematic monitoring system of tobacco use and other health behaviours in our country.

\section{CONCLUSIONS}

The tobacco control efforts should be focused on the population of Poles at large. However, according to the results of our analysis, the most important, modifiable factors that are related to heavy smoking include early age of smoking onset and a lack of awareness of the adverse effects of tobacco use.

\section{Recommendations for future tobacco control activities}

The results of GATS in Poland indicate that measures intended to prevent starting smoking by children and young people should have the highest priority. In this light, tobacco control initiatives should focus on deglamorization and denormalization of smoking, limiting the availability of tobacco products through consistent compliance with the prohibition of sales to minors, and fiscal policy [27]. Effective enforcement of bans on smoking in educational establishments and public places is necessary as well $[28,29]$. Moreover, comprehensive bans on tobacco advertising, the disclosure of tobacco products at point of sales, limitation and highlighting dishonest tactics to the public, of fake corporate social responsibility programs, such as Stop 18, which does not aim to reduce smoking but to increase social acceptance and perception of tobacco companies as the company socially responsible, should be applied. Ban on extending the brand into products not associated with tobacco, for example clothing, seems to be also an important step to decrease approval for, and attractiveness of tobacco products to young people.

To raise awareness of the adverse health effects of smoking; of key importance are educational activities implemented within the framework of teaching as well as local, national or global mass media campaigns. Pictorial warnings on cigarette packs are also an essential component of elevating awareness of the harmfulness of tobacco products. It is a proven effective strategy; therefore, pictorial warnings should be introduced as soon as possible in Poland.

We should also remember about the people who are already smokers. Smokers, not only heavy smokers, should be encouraged to quit smoking and supported when they decide to do so.

For those who require specialist service (individual, group, telephone-based counselling), there is a need to ensure the availability of medical staff and therapists. There is a general perception that health professionals are not engaged in smoking cessation. This is partly due to the very low incentive given by the health-care system to the health-care providers, and partly to insufficient training in the treatment of tobacco dependence. Furthermore, tobacco dependence medication should be more affordable and offered in public health care centres free of charge or at least partly reimbursed. More quit lines would be a decisive and tangible support to smokers who want to quit. Strengthening the existing national quit line and coordinating the integration of national and local quit lines, which should be appropriately staffed and funded, and be accessible on a full-time basis is necessary. Easy access to tailored print or web-based cessation materials should be assured too. Although morbidity and mortality from tobacco-related diseases are among the highest in the world, there is still a lack of comprehensive cessation support for smokers in our country [17]. 


\section{ACKNOWLEDGEMENTS}

Financial support was provided by the Bloomberg Initiative to Reduce Tobacco Use, a program of Bloomberg Philanthropies. We would like to thank the representatives of the Global Adult Tobacco Survey Scientific Committee (Poland) including: Professor Witold Zatoński (Cancer Centre and Institute of Oncology, Warszawa), Professor Bolesław Samoliński (Medical University, Warszawa), Dr. Przemysław Biliński (Chief Sanitary Inspector, Warszawa), and members of the Technical and Survey Staff Team as well as our Partners from the World Health Organization, Centers for Disease Control and Prevention, CDC Foundation, Johns Hopkins Bloomberg, School of Public Health and the RTI International for their collaboration and support.

\section{REFERENCES}

1. WHO Report on the Global Tobacco Epidemic, 2008. The MPOWER package. Geneva: WHO; 2008.

2. World Health Organization. Global Status report on noncommunicable diseases 2010. Geneva: WHO; 2011.

3. World Health Organization (WHO). The current status of the tobacco epidemic in Poland. Copenhagen: WHO; 2009.

4. Lubin JH, Alavanja MC, Caporaso N, Brown LM, Brownson RC, Field RW, et al. Cigarette smoking and cancer risk: modelling total exposure and intensity. Am J Epidemiol 2007;166(4):479-89.

5. Lubin J, Virtamo J, Weinstein S, Albanes D. Cigarette Smoking and Cancer: Intensity Patterns in the Alpha-Tocopherol, Beta-Carotene Cancer Prevention Study in Finnish Men. Am J Epidemiol 2008; 167(8):970-5. DOI: 10.1093/aje/kwm392.

6. Lynch SM, Vrieling A, Lubin JH, Kraft P, Mendelsohn JB, Hartge P, et al. Cigarette smoking and pancreatic cancer: a pooled analysis from the pancreatic cancer cohort consortium. Am J Epidemiol 2009;Aug 15;170(4):403-13.

7. Strandberg AY, Strandberg TE, Pitkala K., Salomaa VV, Tilvis RS, Miettinen TA. The effect of smoking in midlife on health-related quality of life in old age. Arch Intern Med 2008;168(18):1986-74.
8. Killen J, Fortmann S, Telch M, Newman B. Are heavy smokers different from light smokers? A comparison after 48 hours without cigarettes. JAMA 1988; 260(11):1581-5.

9. Wilson D, Wakefield M, Owen N, Roberts L. Characteristics of heavy smokers. Prev Med 1992;21(3):311-9.

10. World Health Organization (WHO). Systematic review of the link between tobacco and the poverty. WHO 2011, Geneva.

11. Kaleta D, Kozieł A, Miśkiewicz P. Global Adult Tobacco Survey in Poland - the aim and current experiences. Med Pr 2009;60(3):197-200 [in Polish].

12. Warren CW, Lee J, Lea V, Goding A, O'Hara B, Carlberg M, et al. Evolution of the Global Tobacco Surveillance System (GTSS) 1998-2008. Global Health Promotion 2009; 16 (Suppl2):4-37.

13. Warren CW, Asma S, Lee J, Lea V, Mackay J. Global Tobacco Surveillance System. The GTSS Atlas. Atlanta: CDC Fundation; 2009.

14. Baumert J, Ladwig KH, Ruf E, Meisinger C, Döring A, Wichmann HE. Determinants of Heavy Cigarette Smoking: Are There Differences in Men and Women? Results From the Population-Based MONICA/KORA Augsburg Surveys. Nicotine Tob Res 2010;12(12):1220-7.

15. Wilson D, Taylor A, Roberts L. Can we target smoking groups more effectively? A study of male and female heavy smokers. Prev Med 1995;24(4)363-8.

16. Sorensen G, Goldberg RJ, Ockene JK, Klar JM, Tannenbaum T, Lemeshow S. Heavy smoking among a sample of employed women. Am J Prev Med 1992;8:207-14.

17. Sieminska A, Buczkowski K, Jassem E, Lewandowska K, Ucinska R, Chelminska M. Patterns of motivations and ways of quitting smoking among Polish smokers: a questionnaire study. BMC Public Health 2008;Aug 4;8:274.

18. Stead LF, Perera R, Bullen C, Mant D, Lancaster T. Nicotine replacement therapy for smoking cessation. Cochrane Database Syst Rev 2008;Jan 23;(1):CD000146.

19. Goldberg RJ, Ockene KJ, Kristeller JL, Kalan LK, Landon J, Hosmer DW. Factors associated with heavy smoking among 
men and women: the physician-delivered smoking intervention project. Am Heart J 1993;125(3):818-23.

20. Lopez A, Collishaw N, Piha T. A descriptive model of the cigarette epidemic in developed countries. Tob Control 1994;3: 242-7.

21. Kaleta D, Polańska K, Jegier A. Smoking predictors among economically active individuals. Int J Occup Med Environ Health 2007;20(4):357-63.

22. Kaleta D, Jegier A. Predictors of inactivity in the working-age population. Int J Occup Med Environ Health 2007;20(2): $175-82$.

23. West R, Zatonski W, Przewozniak K, Jarvis MJ. Can we trust national smoking prevalence figures? Discrepancies between biochemically assessed and self-reported smoking rates in three countries. Cancer Epidemiol Biomarkers Prev 2007;16(4):820-2.

24. Kentalaa J, Utriainenb P, Pahkalac K, Mattila K. Verification of adolescent self-reported smoking. Addict Behav 2004;29:405-11.
25. Patrick DL, Cheadle A, Thompson DC, Diehr P, Koepsell T, Kinne S. The Validity of Self-Reported Smoking: A Review and Meta-Analysis. Am J Public Health 1994;84: 1086-93.

26. Polakowska M, Piotrowski W, Tykarski A, Drygas W, Wyrzykowski B, Pajak A, et al. Addiction to tobacco smoking in the Polish population. Results of the WOBASZ program. Kardiol Pol 2005;63(Suppl 4):626-31 [in Polish].

27. Kaleta D, Usidame B, Polańska K. Tobacco advertisements to women: creating an awareness among women. Cent Eur J Public Health 2011;19(2):73-8.

28. Kaleta D, Polańska K, Wojtysiak P, Kozieł A, Kwaśniewska M, Miśkiewicz $\mathrm{P}$, et al. Effective protection from exposure to environmental tobacco smoke in Poland: The World Health Organization perspective. Int J Occup Med Environ Health 2010;23(2):123-31.

29. World Health Organization (WHO). WHO report on the global tobacco epidemic. Implementing smoke-free environments. Geneva: WHO; 2009.

This work is available in Open Access model and licensed under a Creative Commons Attribution-NonCommercial 3.0 Poland License - http://creativecommons.org/ licenses/by-nc/3.0/pl/deed.en. 